\title{
Radiative Transfer in Combustion Processes
}

\author{
P. J. Coelho \\ Mechanical Engineering Department \\ Instituto Superior Técnico, Universidade de Lisboa \\ Av. Rovisco Pais, 1049-001 Lisboa, Portugal \\ pedro.coelho@tecnico.ulisboa.pt
}

Thermal radiation plays a key role in many combustion processes, including laboratory flames and combustors, industrial furnaces and boilers, and fires. However, the numerical simulation of combustion processes often overlooks the importance of thermal radiation, and employs crude approximations that may compromise the accuracy of the results. This is partly due to the need to reduce the computational time spent in the calculation of radiative transfer, but also due to other reasons, such as the use of commercial software where state of the art models are not available, or lack of knowledge of the merits and limitations of the models employed. In this lecture, an overview of the models available to solve the radiative transfer equation, to calculate the radiative properties of the medium and to account for the interaction between turbulence and combustion is presented, and recommendations for practical applications are given. 